\title{
CALENDAR
}

\section{November 2000}

\section{Surviving Cancer - Conference}

St Luke's (Cheshire) Hospice, Winsford, Cheshire, UK

\section{Further information from:}

Cheshire Hospices Education, Queensway, Winsford, Cheshire CW7 4AW, UK. Tel: + 44 (0) 1606 559292; Fax: + 44 (0) 1606 553696; E-mail: info@che.org.uk; Website: www.che.org.uk/ eduhosp

\section{1-13 December 2000}

\section{Genes \& Cancer 2000}

UK Molecular Biology \& Cancer Network Meeting XVII University of Warwick, UK

\section{Further information from}

Transcriptional Control, Cytoplasmic Signalling, Nuclear Structures, Cell Cycle Control, Therapeutics

Dr Stefan Roberts, Department of Biochemistry, University of Dundee, Dundee, UK. Tel: + 44 (0) 1382 344248;

Fax: + 44 (0) 1382 345783; E-mail: s.g.e.roberts@dundee.ac.uk; Website: www.icr.uk/ukmbcn/info.htm

\section{5-7 February 2001}

\section{3rd UICC Cancer Management Meeting - Quality} Cancer Care in the New Millennium

Republic of Singapore

Further information from:

Secretariat, The National Cancer Centre (Singapore), 11 Hospital Drive Level 4, Singapore 169610, Republic of Singapore. Tel: + 65436 8294/8283/8186; Fax: + 65225 7559; E-mail: 3rduice@nccs.com.sg; Website: www.nccs.com.sg

\section{4-17 February 2001}

\section{International Conference on Dietary Factors: Cancer Causes \& Prevention \\ Vienna, Austria}

The following main areas will be covered:

1. Chemical analytical data on hazardous and protective constituents of foods

2. Mutagens and antimutagens in the human diet
3. Post initiation effects of dietary factors

4. Human biomonitoring and risk assessment

5. Epidemiology, trends in nutrition and dietary recommendations

Further information from:

Johanna B. Baukovits, Institute for Cancer Research, Borschkegasse 8a, A-1090 Vienna, Austria. Fax: + 431427796

51; E-mail: walter.paukovits@univie.ac.at

\section{7-10 March 2001}

4th International Symposium on Leukemia and Lymphoma - molecular pharmacology and new treatment modalities

Amsterdam, The Netherlands

Chairman: G.J.L. Kaspers, R. Pieters, P. Sonneveld and A.J.P. Veerman

Abstract deadline: 1 December 2000

Further information from:

VU Conference Service, De Boelelaan 1105, NL-1081 HV

Amsterdam, The Netherlands. Tel: + 31 (0) 20444 5790;

Fax: + 31 (0) 20444 5825; E-mail: vu conference@dienst.vu.nl

21-23 May 2001

\section{UK Radiological Congress 2001 (UKRC 2001)} (Incorporating IOS \& MED X RAY ${ }^{\circledR}$ )

Wembley Conference \& Exhibition Centre, London, UK

Further information from:

UKRC Secretariat, PO Box 2895, London W1A 5RS, UK. Tel: + 44 (0) 207307 1410/20; Fax: + 44 (0) 207307 1414; E-mail: ukrc@dial.pipex.com; Website: www.ukrc.org.uk

\section{5-16 March 2002}

4th International Conference on the Adjuvant Therapy of Malignant Melanoma

The Royal College of Physicians, London

Further information from:

Conference Secretariat, CCI Ltd, 2 Palmerston Court, Palmerston Way, London SW8 4AJ, UK. Tel: + 44 (0) 207720 0600; Fax: + 44 (0) 20 7720 7177; E-mail: melanoma@confcomm.co.uk; Website: www.confcomm.co.uk/Melanoma 